\title{
ERROR BOUNDS DUE TO RANDOM NOISE IN CYLINDRICAL NEAR-FIELD MEASUREMENTS
}

J. Romeu, L. Jofre

\section{ABSTRACT.}

The far field errors due to near field random noise are statistically bounded when performing cylindrical near to far field transform. In this communication, the far field noise variance it is expressed as a function of the measurement parameters and the near field noise variance.

\section{INTRODUCTION.}

So far [1] [2], expressions have been derived to predict the effect of random errors in planar near field antenna measurements. Similar equations are not available for cylindrical near field measurements. These expressions relate the near field $S / N$ ratio to the far field $S / N$ ratio. Although they provide good estimates, they are not valid for the cylindrical case. Moreover, the far field $\mathrm{S} / \mathrm{N}$ ratio, although being a quite appealing parameter, hides in its simplicity some important aspects.

The far field noise due to a random near field noise is an stochastic process, and it will be shown that for a white gaussian space stationary near field noise, the far field noise is a gaussian non-stationary in elevation and coloured in azimuth process, with a variance dependent on the measurement probe.

The full characterization of the far field noise allows to compute a far field pattern upper and lower bound for a given probability, giving a deeper understanding of the effect of a random error in the radiation pattern. This is of utmost interest in low side lobe antenna measurements, where random errors become a limiting factor in the final accuracy. The validity of this formulation has been checked with actual measurements.

\section{EAR EIELD TRANSFORMATION OF THE NEAR FIELD NOISE.}

The cylindrical near to far field transformation is based on obtaining the cylindrical modal coefficients of the fields, from the measurement on a cylinder that encloses the Antenna Under Test (AUT). The formulation is well described in [3]. Since the transformation is a linear operator, the effect of an additive noise can be studied by superposition.

The cylindrical modal coefficients of the radiated fields of the AUT are found as:

Universitat Politécnica de Catalunya, Aptat. 30002, 08080 Barcelona, SPAIN. 
$a_{n}\left(k_{z}\right)=-\frac{k \eta}{16 \pi^{2} k_{p}^{2}} \frac{\left[\tilde{v}_{1}\left(n, k_{z}\right) b_{n}^{(2)}\left(-k_{z}\right)-\tilde{v}_{2}\left(n, k_{z}\right) b_{n}^{(1)}\left(-k_{z}\right)\right]}{\Delta\left(n, k_{z}\right)}$

$b_{n}\left(k_{z}\right)=-\frac{k \eta}{16 \pi^{2} k_{p}^{2}} \frac{\left[\tilde{v}_{2}\left(n, k_{z}\right) a_{n}^{(1)}\left(-k_{z}\right)-\tilde{v}_{1}\left(n, k_{z}\right) a_{n}^{(2)}\left(-k_{z}\right)\right]}{\Delta\left(n, k_{z}\right)}$

with

$$
\Delta\left(n, k_{z}\right)=a_{n}^{(1)}\left(-k_{z}\right) b_{n}^{(2)}\left(-k_{z}\right)-a_{n}^{(2)}\left(-k_{z}\right) b_{n}^{(1)}\left(-k_{z}\right)
$$

and

$$
\begin{aligned}
& a_{n}^{(1) \cdot(2)}\left(-k_{z}\right)=\sum_{m=-\infty}^{\infty} c_{m}^{(1) \cdot(2)}\left(-k_{z}\right) H_{m+n}^{(2)}\left(k_{p} \rho_{0}\right) \\
& b_{n}^{(1) \cdot(2)}\left(-k_{z}\right)=\sum_{m=-\infty}^{\infty} d_{m}^{(1) \cdot(2)}\left(-k_{z}\right) H_{m+n}^{(2)}\left(k_{p} \rho_{0}\right)
\end{aligned}
$$

where $\tilde{v}_{1}$ and $\quad \tilde{v}_{2}$ are the Fourier transforms of the voltage at the measurement probes. The Fourier transform being defined as

$$
\tilde{v}_{c a}\left(\rho=\rho_{0} ; n, k_{z}\right)=\frac{1}{2 \pi} \int_{0}^{2 \pi} \int_{--}^{-} v_{c a}\left(\rho=\rho_{0}, \phi, z\right) e^{-j n \phi} e^{j k z z} d \phi d k_{z}
$$

And $c_{m}^{(1)}, d_{m}^{(1)}$ and $c_{m}^{(2)}, d_{m}^{(2)}$ are the modal coefficients corresponding to the expansion of the probes radiated fields in cylindrical modes.

Making use of the following asymptotic expressions of the far fields, the far field is obtained.

$$
\begin{aligned}
& E_{\theta}=-2 k \operatorname{sen} \theta \frac{e^{-j k r}}{r} \sum_{n=0}^{\mid n<k} b_{n}(k \cos \theta) j^{n+1} e^{j n \phi} \\
& E_{\phi}=j 2 k \operatorname{sen} \theta \frac{e^{-j k r}}{r} \sum_{n=0}^{\left|n<k_{p} \alpha\right|} a_{n}(k \cos \theta) j^{n+1} e^{j n \phi}
\end{aligned}
$$

Let $n(z, \phi)$ be a gaussian white space stationary noise with a variance $\sigma^{2}$. Receiver noise responds to this model. When the near to far field transformation is applied to this kind of noise, the far field noise is a white gaussian noise, non stationary in $\theta$ and coloured in $\phi$. It can be proved that the far field noise variance for each field component is

$$
\begin{aligned}
& \sigma_{f f \theta}^{2}\left(k_{z}, \phi\right)=\sigma^{2}\left[\frac{k \eta}{8 \pi^{2} k_{\rho}}\right]^{2} N_{z} N_{\phi}\left(\frac{\Delta \phi \Delta z}{2 \pi}\right)^{2} \cdot \\
& \cdot \sum_{n} \frac{\left|a_{n}^{(1)}\left(-k_{z}\right)\right|^{2}+\left|a_{n}^{(2)}\left(-k_{z}\right)\right|^{2}}{\left|a_{n}^{(1)}\left(-k_{z}\right) b_{n}^{(2)}\left(-k_{z}\right)-a_{n}^{(2)}\left(-k_{z}\right) b_{n}^{(1)}\left(-k_{z}\right)\right|^{2}}
\end{aligned}
$$




$$
\begin{aligned}
& \sigma_{f f \phi}^{2}\left(k_{z}, \phi\right)=\sigma^{2}\left[\frac{k \eta}{8 \pi^{2} k_{p}}\right]^{2} N_{z} N_{\cdot}\left(\frac{\Delta \phi \Delta z}{2 \pi}\right)^{2} \cdot \\
& \cdot \sum_{n} \frac{\left|b_{n}^{(1)}\left(-k_{z}\right)\right|^{2}+\left|b_{n}^{(2)}\left(-k_{z}\right)\right|^{2}}{\left|a_{n}^{(1)}\left(-k_{z}\right) b_{n}^{(2)}\left(-k_{z}\right)-a_{n}^{(2)}\left(-k_{z}\right) b_{n}^{(1)}\left(-k_{z}\right)\right|^{2}}
\end{aligned}
$$

where $N_{z}$ and $N_{\phi}$, and $\Delta z$ and $\Delta \phi$ are the number of sample points and measurement increment of the near field data. On the other hand, $k_{z}=k \cos \theta$, and $a_{n}^{(i)}\left(k_{z}\right)$ and $b_{n}^{(i)}\left(k_{z}\right)$ are related to the measurement probe cylindrical coefficients [3] [4].

From the expressions above the effect of an additive gaussian white noise in the near field is an additive gaussian noise with variance proportional to the near field noise variance and function of $\mathbf{K z}$, therefore it is non-stationary in $\mathrm{Kz}$. Moreover the variance depends on the probe cylindrical coefficients (radiation pattern), so the effects of the noise will be dependent on the probe and in general different for each polarization, and function of the elevation angle.

The near field variance $\sigma^{2}$ can be estimated from the power content in the evanescent region of the spectral domain, that is assumed to be completely due to the near field random noise.

\section{EAR EIELD BOUNDS.}

The noise contaminated radiation pattern will be of the form

$$
\vec{E}\left(k_{z}, \phi\right)=\vec{E}\left(k_{z}, \phi\right)+\vec{n}\left(k_{z}, \phi\right)
$$

where $\vec{n}\left(k_{z}, \phi\right)$ is a gaussian noise with variance $\sigma_{f f}^{2}\left(k_{z}\right)$ given by equations (7) and (8). The far field module is for a given polarization

$$
|E|=|\Re(E+n)+j \mathfrak{I}(E+n)|=\left(\Re(E+n)^{2}+\mathfrak{I}(E+n)^{2}\right)^{\frac{1}{2}}=\left(X^{2}+Y^{2}\right)^{\frac{1}{2}}
$$

where $\mathrm{X}$ and $\mathrm{Y}$ are the real and imaginary parts of $E\left(k_{z}, \phi\right)+n\left(k_{z}, \phi\right) . \mathrm{X}$ and $\mathrm{Y}$ are gaussian random variables with mean value $R(E)$ and $I(E)$ variance $\sigma_{r e}^{2}$ and $\sigma_{i m}^{2}$ respectively, and

$$
\sigma_{r e}^{2}=\sigma_{i m}^{2}=\frac{1}{2} \sigma_{f f}^{2}
$$

$|E|$ is in this case a random variable with a Rice probability density function (PDF) given by [5]

$$
f(|\tilde{E}|)=\frac{|\tilde{E}|}{\sigma_{r e}^{2}} e^{-\frac{|E|^{2} \cdot|E|^{2}}{20_{r e}^{2}}} I_{0}\left(\frac{|E||E|}{\sigma_{r e}^{2}}\right)
$$

Knowing the PDF of the module of the far field, an upper and lower bound $\mathrm{M}$ and $\mathrm{m}$ for $\mid \tilde{E}$ | can be calculated by 


$$
\begin{gathered}
P(|\tilde{E}| \leq M)=\int_{0}^{M} f|\tilde{E}| d|\tilde{E}|=p \\
P(|\tilde{E}|>m)=1-\int_{0}^{m} f|\tilde{E}| d|\tilde{E}|=q
\end{gathered}
$$

where $p$ and $q$ are the probabilities to exceed the bound $M$ and not to exceed $m$ respectively. The probability that the far field is between $M$ and $m$ is

$$
P(m<|\tilde{E}| \leq M)=\int_{m}^{M} f|\tilde{E}| d|\tilde{E}|=p+q-1
$$

\section{RESULTS.}

Numerical simulations, as well as measurements, have been carried out to validate expressions (7) and (8). The measurements have been done in the system described in [6]. Figure 1 shows the far field variance to near field variance ratio. The probe is an ideal magnetic probe, the frequency is $3 \mathrm{GHz}$ and the measurement radius is $50.9 \mathrm{~cm}$. By an ideal magnetic probe it is understood a probe that responds with a voltage equal to the magnetic field intensity in one point, and only to one polarization. The figure compares the result from equations (7) and (8) and a numerical evaluation of the far field variance which is done by performing a high number of near to far field transformations (1000 in this case) with only noise. Both curves completely agree. It is important to notice that the behaviour of the far field variance is more complex than it could be expected.

The following figures show the radiation pattern of a low sidelobe array. Figures 2 and 3 without noise, and figures 4 and 5 with a $40 \mathrm{~dB}$ S/N ratio in the near field measurement. The dotted lines show the $90 \%$ probability upper and lower bound. Therefore the probability that the noise contaminated far field is between both bounds is a $80 \%$ In this case the result is a numerical simulation and shows that the formulation predicts with good accuracy the effect of random errors in the measurement.

Figure 6 shows the result on a real measurement. In this case, it has been taken a real measurement of a low side lobe antenna with a near field $\mathrm{S} / \mathrm{N}>50 \mathrm{~dB}$ and have added noise so the $\mathrm{S} / \mathrm{N}$ is $40 \mathrm{~dB}$. The figure shows the maximum and minimum error bounds ( $90 \%$ probability) and the actual error when the $S / N$ is worsen to $40 \mathrm{~dB}$. Once again the computed bounds give a good prediction of the actual accuracy of the measurement system.

\section{CONCLUSIONS.}

A full characterization of the far field noise due to near field random white gaussian errors is obtained. Once the statistical properties of the noise are known, an upper and lower bound of the transformed far field are computed. The results have been proved accurate to predict the performance of a real measurement system.

\section{REFERENCES.}

[1] A.C.Newel,C.F. Stubenrauch.'Effect of Random Errors in Planar Near-Field Measurement.' IEEE trans. Antennas Propagat., vol.AP-36, June 1988. 
[2] J.B. Hoffman, K.R. Grimm. 'Far-Field Uncertainty Due to Random Near-Field Measurement Error.' IEEE Trans. Antennas Propagat., vol. AP-36, June 1988.

[3] W.M. Leach, D.T. Paris, 'Probe Compensated Near-Field Measurements on a Cylinder.' IEEE Trans. Antennas Propagat., vol. AP-21, July 1973.

[4] G.V. Borgiotti 'Integral Equation Formulation for Probe Corrected Far-Field Reconstruction from Measurements on a Cylinder.' vol. AP-26, July 1978.

[5] D.O. North, 'An Analysis of the Factors Which Determine Signal/Noise Discrimination in Pulsed-Carrier Systems' Proc. IEEE, 51, July 1963, pp. 1016-1027.

[6] J. Romeu, M. Baquero, M. Ferrando, L. Jofre, J. Alemany, V. Gonzalez, 'A Cylindrical Near Field Test Facility', Microwave Engineering Europe, September/October 1990, pp. 25-31.

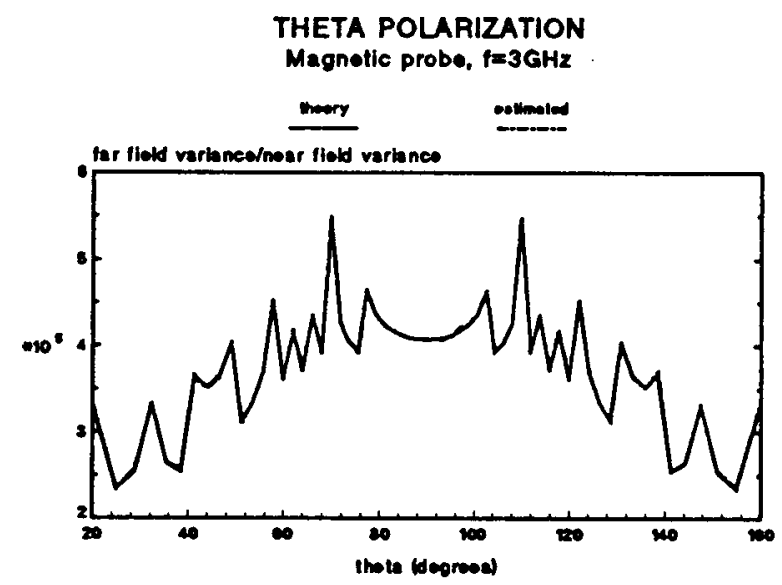

Figure 1. Far field variance for a magnetic probe, as a function of elevation angle.

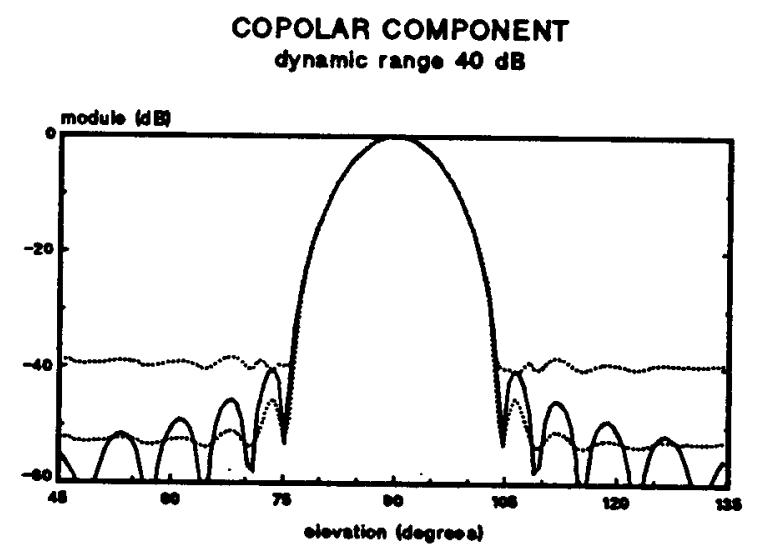

Figure 2. Simulated radiation pattern and upper and lower bounds for a $S / N=40 \mathrm{~dB}$, copolar component. 
CROSSPOLAR COMPONENT dynamic range $40 \mathrm{~dB}$

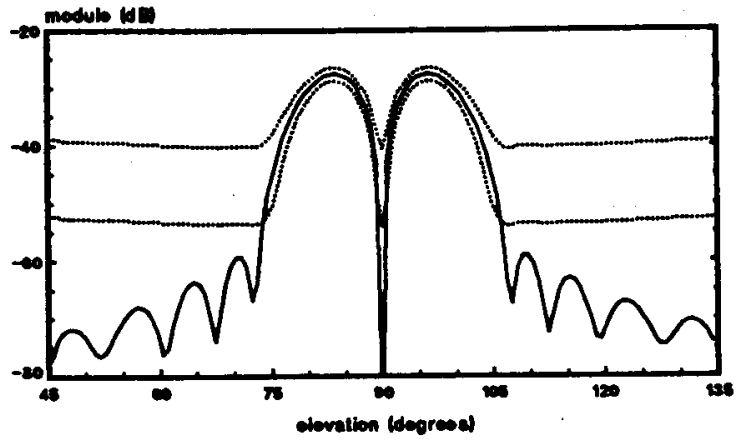

Figure 3. Simulated radiation pattern and upper and lower bounds for a $S / N=40 \mathrm{~dB}$, crossolar component.

CROSSPOLAR COMPONENT dynamic range $40 \mathrm{~dB}$

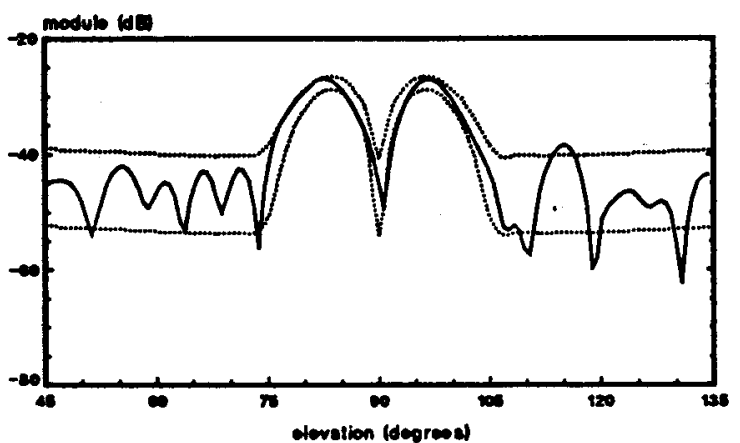

Figure 5. Simulated radiation pattern as in figure 3, but with a near field $S / N=40$ dB.
COPOLAR COMPONENT

dynamic range $40 \mathrm{~dB}$

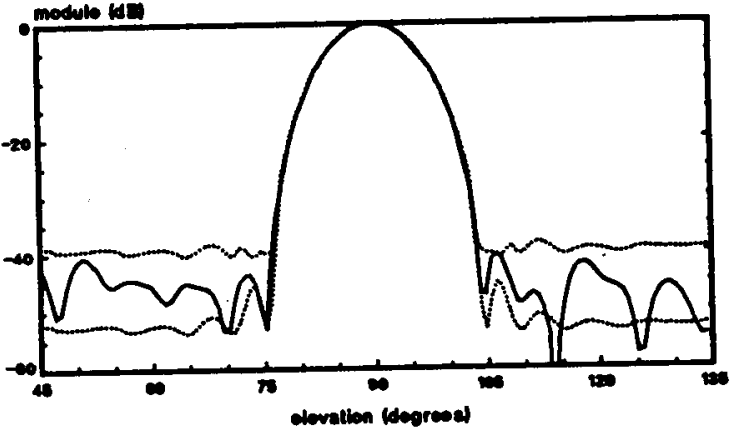

Figure 4. Simulated radiation pattern as in figure 2, but with a near field $S / N=40 \mathrm{~dB}$.

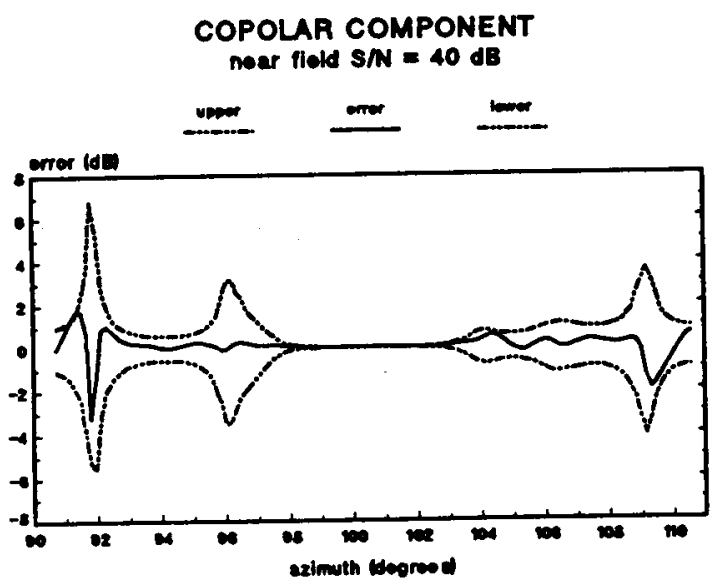

Figure 6. Error bounds and radiation pattern error on a real low side lobe measurement. 